J. Nonlinear Var. Anal. 6 (2022), No. 1, pp. 51-67

Available online at http://jnva.biemdas.com

https://doi.org/10.23952/jnva.6.2022.1.04

\title{
AN INERTIAL NON-MONOTONIC SELF-ADAPTIVE ITERATIVE ALGORITHM FOR SOLVING EQUILIBRIUM PROBLEMS
}

\author{
HABIB UR REHMAN ${ }^{1}$, POOM KUMAM $^{1,2, *}$, YEKINI SHEHU ${ }^{3}$, MURAT OZDEMIR $^{4}$, WIYADA KUMAM ${ }^{5}$ \\ ${ }^{1}$ Departments of Mathematics, Faculty of Science, \\ King Mongkut's University of Technology Thonburi (KMUTT), Bangkok 10140, Thailand \\ ${ }^{2}$ Department of Medical Research, China Medical University Hospital, \\ China Medical University, Taichung 40402, Taiwan \\ ${ }^{3}$ Department of Mathematics, Zhejiang Normal University, Jinhua 321004, China \\ ${ }^{4}$ Department of Mathematics, Ataturk University, Erzurum 25030, Turkey \\ ${ }^{5}$ Applied Mathematics for Science and Engineering Research Unit (AMSERU), Program in Applied Statistics, \\ Department of Mathematics and Computer Science, Faculty of Science and Technology, \\ Rajamangala University of Technology Thanyaburi (RMUTT), Pathum Thani 12110, Thailand
}

\begin{abstract}
In this paper, we introduce a modification of the extragradient algorithm with a non-monotonic stepsize rule to solve equilibrium problems. This modification is based on the inertial subgradient technique. Under mild conditions, such as, the Lipschitz continuity and the monotonicity of a bifunction (including the pseudomonotonicity), the strong convergence of the proposed algorithm is established in a real Hilbert space. The proposed algorithm uses a non-monotonic stepsize rule based on the local bifunction information rather than its Lipschitz-type constants or other line search methods. We present various numerical examples, which illustrate the strong convergence of the algorithm.
\end{abstract}

Keywords. Equilibrium problem; Inertial method; Lipschitz-type conditions; Non-monotonic stepsize rule; Strong convergence.

\section{INTRODUCTION}

Let $\mathbb{C}$ be a nonempty, convex, and closed subset of a real Hilbert space $\mathbb{H}$. Let $f: \mathbb{H} \times \mathbb{H} \rightarrow \mathbb{R}$ be a bifunction with $f(y, y)=0$ for each $y \in \mathbb{C}$. Recall that the known equilibrium problem is defined in the following way: Find $\wp^{*} \in \mathbb{C}$ such that

$$
f\left(\wp^{*}, y\right) \geq 0, \forall y \in \mathbb{C} .
$$

This problem draw much attention due to the facts that it has lots of real applications and includes a number of mathematical problems, such as fixed point problems, vector and scalar minimization problems, variational inequalities, complementarity problems, and saddle point problems as special cases; see, e.g., $[1,2,3,4,5]$ and the references therein. To the best of our knowledge, the term "equilibrium problem" was introduced in 1992 by Muu and Oettli [1] and

${ }^{*}$ Corresponding author.

E-mail address: poom.kum@kmutt.ac.th (P. Kumam).

Received May 29, 2021; Accepted July 11, 2021.

(C)2022 Journal of Nonlinear and Variational Analysis 
further effectively studied by Blum and Oettli [2]. Problem (EP) is also known as the Ky Fan inequality because of his initial contribution to the field [6].

Recently, many researchers introduced and studied various iterative methods for solving problem (EP), such as proximal point methods, extragradient-like methods, and hybrid projection methods; see, e.g., [7, 8, 9, 10, 11, 12] and the references therein. One of useful methods is the famous extragradient method introduced by Flam et al. [13] and Tran et al. [10]. The method reads as follows. Select an arbitrary starting point $u_{0} \in \mathbb{H}$. For the current iterate $u_{n}$, we obtain the next iteration $u_{n+1}$ by the following iterative process

$$
\left\{\begin{array}{l}
u_{n} \in \mathbb{C}, \\
y_{n}=\underset{y \in \mathbb{C}}{\arg \min }\left\{\zeta f\left(u_{n}, y\right)+\frac{1}{2}\left\|u_{n}-y\right\|^{2}\right\}, \\
u_{n+1}=\underset{y \in \mathbb{C}}{\arg \min }\left\{\zeta f\left(y_{n}, y\right)+\frac{1}{2}\left\|u_{n}-y\right\|^{2}\right\},
\end{array}\right.
$$

where $c_{1}$ and $c_{1}$ are Lipschitz constants of $f_{1}$ and $f_{2}$, and $0<\zeta<\min \left\{\frac{1}{2 c_{1}}, \frac{1}{2 c_{2}}\right\}$. We remark that the extragradient method was first introduced and studied by Korpelevich [14] for solving saddle point problems. It is also important to note that the above results in Flam et al. [13] and Tran et al. [10] used a constant stepsize that is dependent on the Lipschitz-type constants of the bifunction. In [10], a weak convergence result was established. The above methods are limited from the viewpoint of computation because the Lipschitz-type constants are usually unknown or not easy to calculate. Recently, Hieu et al. [11] introduced a new gradient-based method for solving pseudomonotone equilibrium problems with the aid of the new stepsize rule, however, their stepsize sequence is nonincreasing and the results in Hieu et al. [11] may depend on the choice of initial stepsize.

Recently, the inertial-type method were extensively studied to accelerate original algorithms; see, e.g., $[15,16,17,18,19]$ and the references therein. It was initially derived from the oscillator equation with a damping and conservative force restoration. This second-order dynamical system is called a heavy friction ball, which was first studied by Polyak [20]. The main feature of inertial-type methods is that we can use the two previous iterations to obtain the next iteration. Numerical findings confirm that the inertial effect strengthens the efficiency of algorithms; see, e.g., [21, 22] and the references therein. In view of the above results, the following question arises naturally. Can one devise a strongly convergent inertial extragradient-like algorithm with non-monotone stepsize rules to solve problem (EP) that does not depends on the Lipschitz-type constants and contractive mappings?

Motivated by the works of Censor et al. [23] and Hieu et al. [11], we give a positive answer to above question with the aid of inertial subgradient techniques in the context of infinitedimensional real Hilbert spaces. Our main contributions in this paper are listed below. We introduce an inertial subgradient extragradient method with a non-monotone stepsize rule to solve the equilibrium problem in a real Hilbert space. The bifunction in problem (EP) is pseudomonotone. We obtain a strong convergence result without the aid of contractive mappings. Numerical experiments are provided to show that our algorithm is efficient and performs better than the existing ones. 
The rest of the paper is organized as follows. Section 2 includes basic definitions and lemmas. In Section 3, we introduce the new inertial subgradient extragradient algorithm with a nonmonotonic stepsize rule. In Section 4, the last section, we give numerical results to illustrate the behaviour of our algorithm.

\section{PRELIMINARIES}

Let $\mathbb{C}$ be a nonempty, convex, and closed subset of a real Hilbert space $\mathbb{H}$. Let $f: \mathbb{H} \times \mathbb{H} \rightarrow \mathbb{R}$ be a bifunction. For problem (EP), we recall the following definitions (see [2, 24]).

(C1) $f$ is said to be pseudomonotone on $\mathbb{C}$ if $f\left(u_{1}, u_{2}\right) \geq 0 \Longrightarrow f\left(u_{2}, u_{1}\right) \leq 0, \forall u_{1}, u_{2} \in \mathbb{C}$.

(C2) $f$ is said to be Lipschitz-type continuous on $\mathbb{C}$ if there exist two constants $c_{1}, c_{2}>0$ such that $f\left(u_{1}, u_{3}\right) \leq f\left(u_{1}, u_{2}\right)+f\left(u_{2}, u_{3}\right)+c_{1}\left\|u_{1}-u_{2}\right\|^{2}+c_{2}\left\|u_{2}-u_{3}\right\|^{2}, \forall u_{1}, u_{2}, u_{3} \in \mathbb{C}$.

(C3) $\limsup _{n \rightarrow \infty} f\left(u_{n}, y\right) \leq f\left(q^{*}, y\right)$, where $\left\{u_{n}\right\}$ is a sequence weakly converging to $q^{*}$.

(C4) $f(u, \cdot)$ is convex and subdifferentiable on $\mathbb{H}$ for each fixed $u \in \mathbb{H}$, and the solution set $E P(f, \mathbb{C})$ is nonempty.

A metric projection $P_{\mathbb{C}}(u)$ of $u \in \mathbb{H}$ onto a closed and convex subset $\mathbb{C}$ of a Hilbert space $\mathbb{H}$ is defined by $P_{\mathbb{C}}(u)=\arg \min \{\|y-u\|: y \in \mathbb{C}\}$. A normal cone of $\mathbb{C}$ at $u \in \mathbb{C}$ is defined by $N_{\mathbb{C}}(u)=\{z \in \mathbb{H}:\langle z, y-u\rangle \leq 0, \forall y \in \mathbb{C}\}$. Let $\mho: \mathbb{C} \rightarrow \mathbb{R}$ be a convex function. Then, the subdifferential of $\mho$ at $u \in \mathbb{C}$ is defined by $\partial \mho(u)=\{z \in \mathbb{H}: \mho(y)-\mho(u) \geq\langle z, y-u\rangle, \forall y \in \mathbb{C}\}$.

Lemma 2.1. [25] Let $\mho: \mathbb{C} \rightarrow \mathbb{R}$ be a convex, subdifferentiable and lower semi-continuous function on $\mathbb{C}$. An element $u \in \mathbb{C}$ is a minimizer of a function $\mho$ if and only if $0 \in \partial \mho(u)+N_{\mathbb{C}}(u)$, where $\partial \mho(u)$ stands for the subdifferentiable of $\mho$ at $u \in \mathbb{C}$, and $N_{\mathbb{C}}(u)$ stands for the normal cone of $\mathbb{C}$ at $u$.

Lemma 2.2. [26] Let $\left\{\mho_{n}\right\}$ be a sequence of non-negative real numbers such that $\mho_{n+1} \leq(1-$ $\left.b_{n}\right) \mho_{n}+b_{n} \ell_{n}$, where $\left\{b_{n}\right\} \subset(0,1)$ and $\left\{\ell_{n}\right\} \subset \mathbb{R}$ are two sequences such that $\lim _{n \rightarrow \infty} b_{n}=0$, $\sum_{n=1}^{\infty} b_{n}=\infty$, and $\lim \sup _{n \rightarrow \infty} \ell_{n} \leq 0$. Then, $\lim _{n \rightarrow \infty} \mho_{n}=0$.

Lemma 2.3. [27] Assume that $\left\{\mho_{n}\right\}$ is a sequence of real numbers such that there exists a subsequence $\left\{n_{i}\right\}$ of $\{n\}$ such that $\mho_{n_{i}}<\mho_{n_{i+1}}$ for all $i \in \mathbb{N}$. Then, there is a non decreasing sequence $m_{k} \subset \mathbb{N}$ such that $m_{k} \rightarrow \infty$ as $k \rightarrow \infty$, and the following conditions are fulfilled by all (sufficiently large) numbers $k \in \mathbb{N}: \mho_{m_{k}} \leq \mho_{m_{k+1}}$ and $\mho_{k} \leq \mho_{m_{k+1}}$. In fact, $m_{k}=\max \{j \leq k$ : $\left.\mho_{j} \leq \mho_{j+1}\right\}$.

\section{MAin Results}

In this section, we present a subgradient extragradient-like algorithm that combines both the non-monotonic stepsize rule and the inertial term and give strong convergence results in this section. Our algorithm is given as follows.

Algorithm 3.1. Step 0: Initially select $\alpha \geq 0, \zeta_{1}>0, \mu \in(0,1)$ and $u_{0}, u_{1} \in \mathbb{C}$. Moreover, select a non-negative real sequence $\left\{\varphi_{n}\right\}$ such that $\sum_{n=1}^{\infty} \varphi_{n}<+\infty$ and choose $\left\{\beta_{n}\right\} \subset(0,1)$ such that $\lim _{n \rightarrow \infty} \beta_{n}=0$ and $\sum_{n=1}^{\infty} \beta_{n}=+\infty$. 
Step 1: Compute $\chi_{n}=u_{n}+\alpha_{n}\left(u_{n}-u_{n-1}\right)-\beta_{n}\left[u_{n}+\alpha_{n}\left(u_{n}-u_{n-1}\right)\right]$ and choose $\alpha_{n}$ such that

$$
0 \leq \alpha_{n} \leq \hat{\alpha}_{n} \quad \text { and } \quad \hat{\alpha}_{n}= \begin{cases}\min \left\{\alpha, \frac{\varepsilon_{n}}{\left\|u_{n}-u_{n-1}\right\|}\right\} & \text { if } u_{n} \neq u_{n-1}, \\ \alpha & \text { otherwise }\end{cases}
$$

where $\varepsilon_{n}=\circ\left(\beta_{n}\right)$ a positive sequence such that $\lim _{n \rightarrow \infty} \frac{\varepsilon_{n}}{\beta_{n}}=0$.

Step 2: Compute $y_{n}=\underset{y \in \mathbb{C}}{\arg \min }\left\{\zeta_{n} f\left(\chi_{n}, y\right)+\frac{1}{2}\left\|\chi_{n}-y\right\|^{2}\right\}$. If $\chi_{n}=y_{n}$, then STOP and $y_{n}$ is a solution. Otherwise, go to Step 3.

Step 3: Firstly choose $\omega_{n} \in \partial_{2} f\left(\chi_{n}, y_{n}\right)$ satisfying $\chi_{n}-\zeta_{n} \omega_{n}-y_{n} \in N_{\mathbb{C}}\left(y_{n}\right)$ and construct a half-space

$$
\mathbb{H}_{n}=\left\{w \in \mathbb{H}:\left\langle\chi_{n}-\zeta_{n} \omega_{n}-y_{n}, w-y_{n}\right\rangle \leq 0\right\} .
$$

Compute $u_{n+1}=\underset{y \in \mathbb{H}_{n}}{\arg \min }\left\{\zeta_{n} f\left(y_{n}, y\right)+\frac{1}{2}\left\|\chi_{n}-y\right\|^{2}\right\}$.

Step 4: Revise the stepsize $\zeta_{n+1}$ as follows:

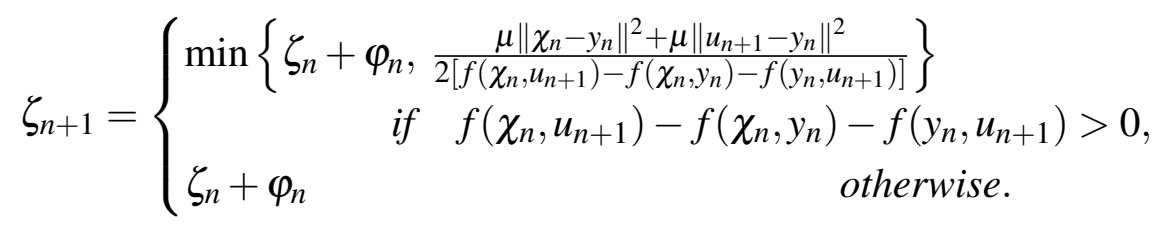

Set $n:=n+1$ and go to Step 1 .

Lemma 3.1. Sequence $\left\{\zeta_{n}\right\}$ converges to $\zeta$ and $\min \left\{\frac{\mu}{\max \left\{2 c_{1}, 2 c_{2}\right\}}, \zeta_{1}\right\} \leq \zeta \leq \zeta_{1}+P$, where $P=\sum_{n=1}^{+\infty} \varphi_{n}$.

Proof. Due to the Lipschitz-type continuity, there exist constants $c_{1}>0$ and $c_{2}>0$. Assume that $f\left(\chi_{n}, u_{n+1}\right)-f\left(\chi_{n}, y_{n}\right)-f\left(y_{n}, u_{n+1}\right)>0$ such that

$\frac{\mu\left(\left\|\chi_{n}-y_{n}\right\|^{2}+\left\|u_{n+1}-y_{n}\right\|^{2}\right)}{2\left[f\left(\chi_{n}, u_{n+1}\right)-f\left(\chi_{n}, y_{n}\right)-f\left(y_{n}, u_{n+1}\right)\right]} \geq \frac{\mu\left(\left\|\chi_{n}-y_{n}\right\|^{2}+\left\|u_{n+1}-y_{n}\right\|^{2}\right)}{2\left[c_{1}\left\|\chi_{n}-y_{n}\right\|^{2}+c_{2}\left\|u_{n+1}-y_{n}\right\|^{2}\right]} \geq \frac{\mu}{2 \max \left\{c_{1}, c_{2}\right\}}$.

It follows that

$$
\min \left\{\frac{\mu}{\max \left\{2 c_{1}, 2 c_{2}\right\}}, \zeta_{1}\right\} \leq \zeta_{n} \leq \zeta_{1}+P .
$$

Let $\left[\zeta_{n+1}-\zeta_{n}\right]^{+}=\max \left\{0, \zeta_{n+1}-\zeta_{n}\right\}$ and $\left[\zeta_{n+1}-\zeta_{n}\right]^{-}=\max \left\{0,-\left(\zeta_{n+1}-\zeta_{n}\right)\right\}$. From the definition of $\left\{\zeta_{n}\right\}$, we have

$$
\sum_{n=1}^{+\infty}\left(\zeta_{n+1}-\zeta_{n}\right)^{+}=\sum_{n=1}^{+\infty} \max \left\{0, \zeta_{n+1}-\zeta_{n}\right\} \leq P<+\infty .
$$

That is, $\sum_{n=1}^{+\infty}\left(\zeta_{n+1}-\zeta_{n}\right)+$ is convergent.

Next, we need to prove the convergence of $\sum_{n=1}^{+\infty}\left(\zeta_{n+1}-\zeta_{n}\right)^{-}$. Let $\sum_{n=1}^{+\infty}\left(\zeta_{n+1}-\zeta_{n}\right)^{-}=+\infty$. Hence, $\zeta_{n+1}-\zeta_{n}=\left(\zeta_{n+1}-\zeta_{n}\right)^{+}-\left(\zeta_{n+1}-\zeta_{n}\right)^{-}$. This implies

$$
\zeta_{k+1}-\zeta_{1}=\sum_{n=0}^{k}\left(\zeta_{n+1}-\zeta_{n}\right)=\sum_{n=0}^{k}\left(\zeta_{n+1}-\zeta_{n}\right)^{+}-\sum_{n=0}^{k}\left(\zeta_{n+1}-\zeta_{n}\right)^{-} \text {. }
$$

By letting $k \rightarrow+\infty$ in (3.2), we have $\zeta_{k} \rightarrow-\infty$ as $k \rightarrow \infty$. This is a contradiction. Letting $k \rightarrow+\infty$ in (3.2), we obtain $\lim _{n \rightarrow \infty} \zeta_{n}=\zeta$. This completes the proof. 
Lemma 3.2. Assume that conditions $(\mathrm{C} 1)-(\mathrm{C} 4)$ are satisfied. Then, the sequence $\left\{u_{n}\right\}$ generated by Algorithm 3.1 is bounded.

Proof. From (3.1), we have $\alpha_{n}\left\|u_{n}-u_{n-1}\right\| \leq \varepsilon_{n}, \forall n \in \mathbb{N}$. Due to $\lim _{n \rightarrow \infty}\left(\frac{\varepsilon_{n}}{\beta_{n}}\right)=0$, we obtain

$$
\lim _{n \rightarrow \infty} \frac{\alpha_{n}}{\beta_{n}}\left\|u_{n}-u_{n-1}\right\| \leq \lim _{n \rightarrow \infty} \frac{\varepsilon_{n}}{\beta_{n}}=0
$$

By using Lemma 2.1, we have

$$
0 \in \partial_{2}\left\{\zeta_{n} f\left(y_{n}, \cdot\right)+\frac{1}{2}\left\|\chi_{n}-\cdot\right\|^{2}\right\}\left(u_{n+1}\right)+N_{\mathbb{H}_{n}}\left(u_{n+1}\right) .
$$

For $\omega \in \partial f\left(y_{n}, u_{n+1}\right)$, there exists a vector $\bar{\omega} \in N_{\mathbb{H}_{n}}\left(u_{n+1}\right)$ such that $\zeta_{n} \omega+u_{n+1}-\chi_{n}+\bar{\omega}=0$. It follows that

$$
\left\langle\chi_{n}-u_{n+1}, y-u_{n+1}\right\rangle=\zeta_{n}\left\langle\omega, y-u_{n+1}\right\rangle+\left\langle\bar{\omega}, y-u_{n+1}\right\rangle, \forall y \in \mathbb{H}_{n} .
$$

Since $\bar{\omega} \in N_{\mathbb{H}_{n}}\left(u_{n+1}\right)$ implies that $\left\langle\bar{\omega}, y-u_{n+1}\right\rangle \leq 0$, for all $y \in \mathbb{H}_{n}$, we have

$$
\left\langle\chi_{n}-u_{n+1}, y-u_{n+1}\right\rangle \leq \zeta_{n}\left\langle\omega, y-u_{n+1}\right\rangle, \forall y \in \mathbb{H}_{n} .
$$

Moreover, $\omega \in \partial f\left(y_{n}, u_{n+1}\right)$. Hence,

$$
f\left(y_{n}, y\right)-f\left(y_{n}, u_{n+1}\right) \geq\left\langle\omega, y-u_{n+1}\right\rangle, \forall y \in \mathbb{H} .
$$

Combining (3.3) and (3.4), we obtain

$$
\zeta_{n} f\left(y_{n}, y\right)-\zeta_{n} f\left(y_{n}, u_{n+1}\right) \geq\left\langle\chi_{n}-u_{n+1}, y-u_{n+1}\right\rangle, \forall y \in \mathbb{H}_{n} .
$$

Hence,

$$
\zeta_{n}\left\langle\omega_{n}, u_{n+1}-y_{n}\right\rangle \geq\left\langle\chi_{n}-y_{n}, u_{n+1}-y_{n}\right\rangle .
$$

By using $\omega_{n} \in \partial f\left(\chi_{n}, y_{n}\right)$, we obtain

$$
f\left(\chi_{n}, y\right)-f\left(\chi_{n}, y_{n}\right) \geq\left\langle\omega_{n}, y-y_{n}\right\rangle, \forall y \in \mathbb{H} .
$$

By letting $y=u_{n+1}$, we have

$$
f\left(\chi_{n}, u_{n+1}\right)-f\left(\chi_{n}, y_{n}\right) \geq\left\langle\omega_{n}, u_{n+1}-y_{n}\right\rangle, \forall y \in \mathbb{H} .
$$

Combining (3.6) and (3.7), we arrive at

$$
\zeta_{n}\left\{f\left(\chi_{n}, u_{n+1}\right)-f\left(\chi_{n}, y_{n}\right)\right\} \geq\left\langle\chi_{n}-y_{n}, u_{n+1}-y_{n}\right\rangle .
$$

By substituting $y=\wp^{*}$ into (3.5), we have

$$
\zeta_{n} f\left(y_{n}, \wp^{*}\right)-\zeta_{n} f\left(y_{n}, u_{n+1}\right) \geq\left\langle\chi_{n}-u_{n+1}, \wp^{*}-u_{n+1}\right\rangle .
$$

Observe that $\wp^{*} \in E P(f, \mathbb{C})$ implies $f\left(\wp^{*}, y_{n}\right) \geq 0$. From the pseudomonotonicity of bifunction $f$, we obtain $f\left(y_{n}, \wp^{*}\right) \leq 0$. Thanks to (3.9), we arrive at

$$
\left\langle\chi_{n}-u_{n+1}, u_{n+1}-\wp^{*}\right\rangle \geq \zeta_{n} f\left(y_{n}, u_{n+1}\right) .
$$

From definition of $\zeta_{n+1}$, we obtain

$$
f\left(\chi_{n}, u_{n+1}\right)-f\left(\chi_{n}, y_{n}\right)-f\left(y_{n}, u_{n+1}\right) \leq \frac{\mu\left\|\chi_{n}-y_{n}\right\|^{2}+\mu\left\|u_{n+1}-y_{n}\right\|^{2}}{2 \zeta_{n+1}}
$$


(3.10) and (3.11) yield that

$$
\begin{aligned}
\left\langle\chi_{n}-u_{n+1}, u_{n+1}-\wp^{*}\right\rangle \geq & \zeta_{n}\left\{f\left(\chi_{n}, u_{n+1}\right)-f\left(\chi_{n}, y_{n}\right)\right\} \\
& -\frac{\mu \zeta_{n}}{2 \zeta_{n+1}}\left\|\chi_{n}-y_{n}\right\|^{2}-\frac{\mu \zeta_{n}}{2 \zeta_{n+1}}\left\|u_{n+1}-y_{n}\right\|^{2} .
\end{aligned}
$$

Combining (3.8) and (3.12), we have

$$
\begin{aligned}
\left\langle\chi_{n}-u_{n+1}, u_{n+1}-\wp^{*}\right\rangle \geq & \left\langle\chi_{n}-y_{n}, u_{n+1}-y_{n}\right\rangle \\
& -\frac{\mu \zeta_{n}}{2 \zeta_{n+1}}\left\|\chi_{n}-y_{n}\right\|^{2}-\frac{\mu \zeta_{n}}{2 \zeta_{n+1}}\left\|u_{n+1}-y_{n}\right\|^{2} .
\end{aligned}
$$

Observe that

$$
\begin{gathered}
-2\left\langle\chi_{n}-u_{n+1}, u_{n+1}-\wp^{*}\right\rangle=-\left\|\chi_{n}-\wp^{*}\right\|^{2}+\left\|u_{n+1}-\chi_{n}\right\|^{2}+\left\|u_{n+1}-\wp^{*}\right\|^{2} . \\
2\left\langle y_{n}-\chi_{n}, y_{n}-u_{n+1}\right\rangle=\left\|\chi_{n}-y_{n}\right\|^{2}+\left\|u_{n+1}-y_{n}\right\|^{2}-\left\|\chi_{n}-u_{n+1}\right\|^{2} .
\end{gathered}
$$

Combining (3.13) and (3.14), we have

$$
\left\|u_{n+1}-\wp^{*}\right\|^{2} \leq\left\|\chi_{n}-\wp^{*}\right\|^{2}-\left(1-\frac{\mu \zeta_{n}}{\zeta_{n+1}}\right)\left\|\chi_{n}-y_{n}\right\|^{2}-\left(1-\frac{\mu \zeta_{n}}{\zeta_{n+1}}\right)\left\|u_{n+1}-y_{n}\right\|^{2} .
$$

It follows from the definition of $\left\{\chi_{n}\right\}$ that

$$
\begin{aligned}
\left\|\chi_{n}-\wp^{*}\right\| & =\left\|\left(1-\beta_{n}\right)\left(u_{n}-\wp^{*}\right)+\left(1-\beta_{n}\right) \alpha_{n}\left(u_{n}-u_{n-1}\right)-\beta_{n} \wp^{*}\right\| \\
& \leq\left(1-\beta_{n}\right)\left\|u_{n}-\wp^{*}\right\|+\left(1-\beta_{n}\right) \alpha_{n}\left\|u_{n}-u_{n-1}\right\|+\beta_{n}\left\|\wp^{*}\right\| \\
& \leq\left(1-\beta_{n}\right)\left\|u_{n}-\wp^{*}\right\|+\beta_{n} K_{1}
\end{aligned}
$$

where

$$
\left(1-\beta_{n}\right) \frac{\alpha_{n}}{\beta_{n}}\left\|u_{n}-u_{n-1}\right\|+\left\|\wp^{*}\right\| \leq K_{1} .
$$

It is given that $\zeta_{n} \rightarrow \zeta$, so there exists a fixed number $\mathfrak{I} \in(0,1-\mu)$ such that

$$
\lim _{n \rightarrow \infty}\left(1-\frac{\mu \zeta_{n}}{\zeta_{n+1}}\right)=1-\mu>\mathfrak{I}>0 .
$$

Thus, there exists a fixed finite number $N_{1} \in \mathbb{N}$ such that

$$
\left(1-\frac{\mu \zeta_{n}}{\zeta_{n+1}}\right)>\mathfrak{I}>0, \forall n \geq N_{1}
$$

From (3.15), we rewrite

$$
\left\|u_{n+1}-\wp^{*}\right\|^{2} \leq\left\|\chi_{n}-\wp^{*}\right\|^{2}, \forall n \geq N_{1} .
$$

Combining (3.16) and (3.17), we obtain

$$
\begin{aligned}
\left\|u_{n+1}-\wp^{*}\right\| & \leq\left(1-\beta_{n}\right)\left\|u_{n}-\wp^{*}\right\|+\beta_{n} K_{1} \\
& \leq \max \left\{\left\|u_{n}-\wp^{*}\right\|, K_{1}\right\} \\
& \vdots \\
& \leq \max \left\{\left\|u_{N_{1}}-\wp^{*}\right\|, K_{1}\right\} .
\end{aligned}
$$

This infers that $\left\{u_{n}\right\}$ is a bounded sequence. 
Theorem 3.1. Let $\left\{u_{n}\right\}$ be te sequence generated by Algorithm 3.1, and let (C1)-(C4) be satisfied. Then, $\left\{u_{n}\right\}$ converges strongly to some $\wp^{*}$. Moreover, $P_{E P(f, \mathbb{C})}(0)=\wp^{*}$.

Proof. From (3.16), we have

$$
\begin{aligned}
\left\|\chi_{n}-\wp^{*}\right\|^{2} & \leq\left(1-\beta_{n}\right)^{2}\left\|u_{n}-\wp^{*}\right\|^{2}+\beta_{n}^{2} K_{1}^{2}+2 K_{1} \beta_{n}\left(1-\beta_{n}\right)\left\|u_{n}-\wp^{*}\right\| \\
& \leq\left\|u_{n}-\wp^{*}\right\|^{2}+\beta_{n}\left[\beta_{n} K_{1}^{2}+2 K_{1}\left(1-\beta_{n}\right)\left\|u_{n}-\wp^{*}\right\|\right] \\
& \leq\left\|u_{n}-\wp^{*}\right\|^{2}+\beta_{n} K_{2},
\end{aligned}
$$

for some $K_{2}>0$. Combining (3.15) and (3.18), we obtain

$$
\begin{aligned}
\left\|u_{n+1}-\wp^{*}\right\|^{2} \leq & \left\|u_{n}-\wp^{*}\right\|^{2}+\beta_{n} K_{2}-\left(1-\frac{\mu \zeta_{n}}{\zeta_{n+1}}\right)\left\|\chi_{n}-y_{n}\right\|^{2} \\
& -\left(1-\frac{\mu \zeta_{n}}{\zeta_{n+1}}\right)\left\|u_{n+1}-y_{n}\right\|^{2}
\end{aligned}
$$

Due to the Lipschitz-continuity and pseudomonotonicity of $f$, we have that $\operatorname{EP}(f, \mathbb{C})$ is a closed and convex set (see [10]). Since $\wp^{*}=P_{E P(f, \mathbb{C})}(0)$, we have $\left\langle 0-\wp^{*}, y-\wp^{*}\right\rangle \leq 0$, $\forall y \in E P(f, \mathbb{C})$. The rest of the proof is divided into the following two cases.

Case 1. Assume that there exists a fixed number $N_{2} \in \mathbb{N}\left(N_{2} \geq N_{1}\right)$ such that $\left\|u_{n+1}-\wp^{*}\right\| \leq$ $\left\|u_{n}-\wp^{*}\right\|, \forall n \geq N_{2}$. The above relation implies that $\lim _{n \rightarrow \infty}\left\|u_{n}-\wp^{*}\right\|$ exists. Let $\lim _{n \rightarrow \infty} \| u_{n}-$ $\wp^{*} \|=l$, for some $l \geq 0$. From (3.19) we can write

$$
\left(1-\frac{\mu \zeta_{n}}{\zeta_{n+1}}\right)\left\|\chi_{n}-y_{n}\right\|^{2}+\left(1-\frac{\mu \zeta_{n}}{\zeta_{n+1}}\right)\left\|u_{n+1}-y_{n}\right\|^{2} \leq\left\|u_{n}-\wp^{*}\right\|^{2}+\beta_{n} K_{2}-\left\|u_{n+1}-\wp^{*}\right\|^{2} .
$$

Due to existence of the limit of $\left\|u_{n}-\wp^{*}\right\|$, and the fact that $\beta_{n} \rightarrow 0$, we conclude that

$$
\left\|\chi_{n}-y_{n}\right\| \rightarrow 0 \text { and }\left\|u_{n+1}-y_{n}\right\| \rightarrow 0 \quad \text { as } n \rightarrow \infty .
$$

Hence,

$$
\lim _{n \rightarrow \infty}\left\|\chi_{n}-u_{n+1}\right\| \leq \lim _{n \rightarrow \infty}\left\|\chi_{n}-y_{n}\right\|+\lim _{n \rightarrow \infty}\left\|y_{n}-u_{n+1}\right\|=0 .
$$

Next, we estimate

$$
\begin{aligned}
\left\|\chi_{n}-u_{n}\right\| & =\left\|u_{n}+\alpha_{n}\left(u_{n}-u_{n-1}\right)-\beta_{n}\left[u_{n}+\alpha_{n}\left(u_{n}-u_{n-1}\right)\right]-u_{n}\right\| \\
& \leq \beta_{n} \frac{\alpha_{n}}{\beta_{n}}\left\|u_{n}-u_{n-1}\right\|+\beta_{n}\left\|u_{n}\right\|+\beta_{n}^{2} \frac{\alpha_{n}}{\beta_{n}}\left\|u_{n}-u_{n-1}\right\| \longrightarrow 0
\end{aligned}
$$

which implies that

$$
\lim _{n \rightarrow \infty}\left\|u_{n}-u_{n+1}\right\| \leq \lim _{n \rightarrow \infty}\left\|u_{n}-\chi_{n}\right\|+\lim _{n \rightarrow \infty}\left\|\chi_{n}-u_{n+1}\right\|=0 .
$$

Thus, $\left\{\chi_{n}\right\}$ and $\left\{y_{n}\right\}$ are bounded sequences. The reflexivity of $\mathbb{H}$ and the boundedness of $\left\{u_{n}\right\}$ guarantee that there exists a subsequence $\left\{u_{n_{k}}\right\}$ of $\left\{u_{n}\right\}$ such that $\left\{u_{n_{k}}\right\} \rightarrow \hat{u} \in \mathbb{H}$ as $k \rightarrow \infty$. Next, we prove that $\hat{u} \in E P(f, \mathbb{C})$. From (3.5), we have

$$
\zeta_{n_{k}} f\left(y_{n_{k}}, y\right) \geq \zeta_{n_{k}} f\left(y_{n_{k}}, u_{n_{k+1}}\right)+\left\langle\chi_{n_{k}}-u_{n_{k+1}}, y-u_{n_{k+1}}\right\rangle, \forall y \in \mathbb{H}_{n} .
$$


It follows from (3.11) that

$$
\begin{aligned}
\zeta_{n_{k}} f\left(y_{n_{k}}, u_{n_{k}+1}\right) \geq & \zeta_{n_{k}}\left\{f\left(\chi_{n_{k}}, u_{n_{k}+1}\right)-f\left(\chi_{n_{k}}, y_{n_{k}}\right)\right\}-\frac{\mu \zeta_{n_{k}}}{2 \zeta_{n_{k}+1}}\left\|\chi_{n_{k}}-y_{n_{k}}\right\|^{2} \\
& -\frac{\mu \zeta_{n_{k}}}{2 \zeta_{n_{k}+1}}\left\|u_{n_{k}+1}-y_{n_{k}}\right\|^{2}
\end{aligned}
$$

which implies that

$$
\begin{aligned}
\zeta_{n_{k}} f\left(y_{n_{k}}, y\right) \geq & \zeta_{n_{k}}\left\{f\left(\chi_{n_{k}}, u_{n_{k}+1}\right)-f\left(\chi_{n_{k}}, y_{n_{k}}\right)\right\}+\left\langle\chi_{n_{k}}-u_{n_{k+1}}, y-u_{n_{k+1}}\right\rangle \\
& -\frac{\mu \zeta_{n_{k}}}{2 \zeta_{n_{k}+1}}\left\|\chi_{n_{k}}-y_{n_{k}}\right\|^{2}-\frac{\mu \zeta_{n_{k}}}{2 \zeta_{n_{k}+1}}\left\|u_{n_{k}+1}-y_{n_{k}}\right\|^{2} .
\end{aligned}
$$

where $y$ is an arbitrary element of set $\mathbb{H}_{n}$. From (3.21), (3.22), (3.23), (3.24), and the boundedness of $\left\{u_{n}\right\}$, we conclude that the right-hand side of the above expression goes to zero. In view of $\zeta_{n_{k}}>0$, and $y_{n_{k}} \rightarrow \hat{u}$, we have $0 \leq \limsup _{k \rightarrow \infty} f\left(y_{n_{k}}, y\right) \leq f(\hat{u}, y), \forall y \in \mathbb{H}_{n}$. Hence, $\hat{u} \in E P(f, \mathbb{C})$. Note that

$$
\limsup _{n \rightarrow \infty}\left\langle\wp^{*}, \wp^{*}-u_{n}\right\rangle=\lim _{k \rightarrow \infty}\left\langle\wp^{*}, \wp^{*}-u_{n_{k}}\right\rangle=\left\langle\wp^{*}, \wp^{*}-\hat{u}\right\rangle \leq 0 .
$$

From the fact that $\lim _{n \rightarrow \infty}\left\|u_{n+1}-u_{n}\right\|=0$ and (3.25), we deduce that

$$
\limsup _{n \rightarrow \infty}\left\langle\wp^{*}, \wp^{*}-u_{n+1}\right\rangle \leq \limsup _{n \rightarrow \infty}\left\langle\wp^{*}, \wp^{*}-u_{n}\right\rangle+\limsup _{n \rightarrow \infty}\left\langle\wp^{*}, u_{n}-u_{n+1}\right\rangle \leq 0 .
$$

Observe that

$$
\begin{aligned}
&\left\|\chi_{n}-\wp^{*}\right\|^{2} \\
&=\left\|u_{n}+\alpha_{n}\left(u_{n}-u_{n-1}\right)-\beta_{n} u_{n}-\alpha_{n} \beta_{n}\left(u_{n}-u_{n-1}\right)-\wp^{*}\right\|^{2} \\
&=\left\|\left(1-\beta_{n}\right)\left(u_{n}-\wp^{*}\right)+\left(1-\beta_{n}\right) \alpha_{n}\left(u_{n}-u_{n-1}\right)-\beta_{n} \wp^{*}\right\|^{2} \\
& \leq\left\|\left(1-\beta_{n}\right)\left(u_{n}-\wp^{*}\right)+\left(1-\beta_{n}\right) \alpha_{n}\left(u_{n}-u_{n-1}\right)\right\|^{2}+2 \beta_{n}\left\langle-\wp^{*}, \chi_{n}-\wp^{*}\right\rangle \\
&=\left(1-\beta_{n}\right)^{2}\left\|u_{n}-\wp^{*}\right\|^{2}+\left(1-\beta_{n}\right)^{2} \alpha_{n}^{2}\left\|u_{n}-u_{n-1}\right\|^{2} \\
&+2 \alpha_{n}\left(1-\beta_{n}\right)^{2}\left\|u_{n}-\wp^{*}\right\|\left\|u_{n}-u_{n-1}\right\|+2 \beta_{n}\left\langle-\wp^{*}, \chi_{n}-u_{n+1}\right\rangle+2 \beta_{n}\left\langle-\wp^{*}, u_{n+1}-\wp^{*}\right\rangle \\
& \leq\left(1-\beta_{n}\right)\left\|u_{n}-\wp^{*}\right\|^{2}+\alpha_{n}^{2}\left\|u_{n}-u_{n-1}\right\|^{2}+2 \alpha_{n}\left(1-\beta_{n}\right)\left\|u_{n}-\wp^{*}\right\|\left\|u_{n}-u_{n-1}\right\| \\
&+2 \beta_{n}\left\|\wp^{*}\right\|\left\|\chi_{n}-u_{n+1}\right\|+2 \beta_{n}\left\langle-\wp^{*}, u_{n+1}-\wp^{*}\right\rangle \\
&=\left(1-\beta_{n}\right)\left\|u_{n}-\wp^{*}\right\|^{2}+\beta_{n}\left[\alpha_{n}\left\|u_{n}-u_{n-1}\right\| \frac{\alpha_{n}}{\beta_{n}}\left\|u_{n}-u_{n-1}\right\|\right. \\
&\left.+2\left(1-\beta_{n}\right)\left\|u_{n}-\wp^{*}\right\| \frac{\alpha_{n}}{\beta_{n}}\left\|u_{n}-u_{n-1}\right\|+2\left\|\wp^{*}\right\|\left\|\chi_{n}-u_{n+1}\right\|+2\left\langle\wp^{*}, \wp^{*}-u_{n+1}\right\rangle\right],
\end{aligned}
$$

which together with (3.17) yields that

$$
\begin{aligned}
& \left\|u_{n+1}-\wp^{*}\right\|^{2} \\
& \leq\left(1-\beta_{n}\right)\left\|u_{n}-\wp^{*}\right\|^{2}+\beta_{n}\left[\alpha_{n}\left\|u_{n}-u_{n-1}\right\| \frac{\alpha_{n}}{\beta_{n}}\left\|u_{n}-u_{n-1}\right\|\right. \\
& \left.\quad+2\left(1-\beta_{n}\right)\left\|u_{n}-\wp^{*}\right\| \frac{\alpha_{n}}{\beta_{n}}\left\|u_{n}-u_{n-1}\right\|+2\left\|\wp^{*}\right\|\left\|\chi_{n}-u_{n+1}\right\|+2\left\langle\wp^{*}, \wp^{*}-u_{n+1}\right\rangle\right] .
\end{aligned}
$$


Using (3.22), (3.26), (3.27), and Lemma 2.2, we conclude that $\left\|u_{n}-\wp^{*}\right\| \rightarrow 0$ as $n \rightarrow \infty$.

Case 2: Suppose that there exists a subsequence $\left\{n_{i}\right\}$ of $\{n\}$ such that

$$
\left\|u_{n_{i}}-\wp^{*}\right\| \leq\left\|u_{n_{i+1}}-\wp^{*}\right\|, \forall i \in \mathbb{N} .
$$

From Lemma 2.3, we see that there exists a sequence $\left\{m_{k}\right\} \subset \mathbb{N}$ as $\left\{m_{k}\right\} \rightarrow \infty$ such that

$$
\left\|u_{m_{k}}-\wp^{*}\right\| \leq\left\|u_{m_{k+1}}-\wp^{*}\right\| \quad \text { and }\left\|u_{k}-\wp^{*}\right\| \leq\left\|u_{m_{k+1}}-\wp^{*}\right\| \text {, for all } k \in \mathbb{N} \text {. }
$$

Following Case 1, we see that (3.20) yields

$$
\begin{aligned}
& \left(1-\frac{\mu \zeta_{m_{k}}}{\zeta_{m_{k}+1}}\right)\left\|\chi_{m_{k}}-y_{m_{k}}\right\|^{2}+\left(1-\frac{\mu \zeta_{m_{k}}}{\zeta_{m_{k}+1}}\right)\left\|u_{m_{k}+1}-y_{m_{k}}\right\|^{2} \\
& \leq\left\|u_{m_{k}}-\wp^{*}\right\|^{2}+\beta_{m_{k}} K_{2}-\left\|u_{m_{k}+1}-\wp^{*}\right\|^{2} .
\end{aligned}
$$

Since $\beta_{m_{k}} \rightarrow 0$, we can deduce $\lim _{k \rightarrow \infty}\left\|\chi_{m_{k}}-y_{m_{k}}\right\|=\lim _{k \rightarrow \infty}\left\|u_{m_{k}+1}-y_{m_{k}}\right\|=0$. Hence,

$$
\lim _{k \rightarrow \infty}\left\|u_{m_{k+1}}-\chi_{m_{k}}\right\| \leq \lim _{k \rightarrow \infty}\left\|u_{m_{k+1}}-y_{m_{k}}\right\|+\lim _{k \rightarrow \infty}\left\|y_{m_{k}}-\chi_{m_{k}}\right\|=0 .
$$

Next, we estimate

$$
\begin{aligned}
\left\|\chi_{m_{k}}-u_{m_{k}}\right\| & =\left\|u_{m_{k}}+\alpha_{m_{k}}\left(u_{m_{k}}-u_{m_{k}-1}\right)-\beta_{m_{k}}\left[u_{m_{k}}+\alpha_{m_{k}}\left(u_{m_{k}}-u_{m_{k}-1}\right)\right]-u_{m_{k}}\right\| \\
& \leq \alpha_{m_{k}}\left\|u_{m_{k}}-u_{m_{k}-1}\right\|+\beta_{m_{k}}\left\|u_{m_{k}}\right\|+\alpha_{m_{k}} \beta_{m_{k}}\left\|u_{m_{k}}-u_{m_{k}-1}\right\| \\
& =\beta_{m_{k}} \frac{\alpha_{m_{k}}}{\beta_{m_{k}}}\left\|u_{m_{k}}-u_{m_{k}-1}\right\|+\beta_{m_{k}}\left\|u_{m_{k}}\right\|+\beta_{m_{k}}^{2} \frac{\alpha_{m_{k}}}{\beta_{m_{k}}}\left\|u_{m_{k}}-u_{m_{k}-1}\right\| \longrightarrow 0 .
\end{aligned}
$$

Observe that $\lim _{k \rightarrow \infty}\left\|u_{m_{k}}-u_{m_{k}+1}\right\| \leq \lim _{k \rightarrow \infty}\left\|u_{m_{k}}-\chi_{m_{k}}\right\|+\lim _{k \rightarrow \infty}\left\|\chi_{m_{k}}-u_{m_{k}+1}\right\|=0$. Following Case 1, we have

$$
\limsup _{k \rightarrow \infty}\left\langle\wp^{*}, \wp^{*}-u_{m_{k}+1}\right\rangle \leq 0 .
$$

From (3.27) and (3.28), we have

$$
\begin{aligned}
& \left\|u_{m_{k}+1}-\wp^{*}\right\|^{2} \\
& \leq\left(1-\beta_{m_{k}}\right)\left\|u_{m_{k}}-\wp^{*}\right\|^{2}+\beta_{m_{k}}\left[\alpha_{m_{k}}\left\|u_{m_{k}}-u_{m_{k}-1}\right\| \frac{\alpha_{m_{k}}}{\beta_{m_{k}}}\left\|u_{m_{k}}-u_{m_{k}-1}\right\|\right. \\
& \left.\quad+2\left(1-\beta_{m_{k}}\right)\left\|u_{m_{k}}-\wp^{*}\right\| \frac{\alpha_{m_{k}}}{\beta_{m_{k}}}\left\|u_{m_{k}}-u_{m_{k}-1}\right\|+2\left\|\wp^{*}\right\|\left\|\chi_{m_{k}}-u_{m_{k}+1}\right\|+2\left\langle\wp^{*}, \wp^{*}-u_{m_{k}+1}\right\rangle\right] \\
& \leq \\
& \quad\left(1-\beta_{m_{k}}\right)\left\|u_{m_{k+1}}-\wp^{*}\right\|^{2}+\beta_{m_{k}}\left[\alpha_{m_{k}}\left\|u_{m_{k}}-u_{m_{k}-1}\right\| \frac{\alpha_{m_{k}}}{\beta_{m_{k}}}\left\|u_{m_{k}}-u_{m_{k}-1}\right\|\right. \\
& \left.\quad+2\left(1-\beta_{m_{k}}\right)\left\|u_{m_{k}}-\wp^{*}\right\| \frac{\alpha_{m_{k}}}{\beta_{m_{k}}}\left\|u_{m_{k}}-u_{m_{k}-1}\right\|+2\left\|\wp^{*}\right\|\left\|\chi_{m_{k}}-u_{m_{k}+1}\right\|+2\left\langle\wp^{*}, \wp^{*}-u_{m_{k}+1}\right\rangle\right] .
\end{aligned}
$$

Thus,

$$
\begin{aligned}
& \left\|u_{m_{k}+1}-\wp^{*}\right\|^{2} \\
& \leq\left[\alpha_{m_{k}}\left\|u_{m_{k}}-u_{m_{k}-1}\right\| \frac{\alpha_{m_{k}}}{\beta_{m_{k}}}\left\|u_{m_{k}}-u_{m_{k}-1}\right\|\right. \\
& \left.\quad+2\left(1-\beta_{m_{k}}\right)\left\|u_{m_{k}}-\wp^{*}\right\| \frac{\alpha_{m_{k}}}{\beta_{m_{k}}}\left\|u_{m_{k}}-u_{m_{k}-1}\right\|+2\left\|\wp^{*}\right\|\left\|\chi_{m_{k}}-u_{m_{k}+1}\right\|+2\left\langle\wp^{*}, \wp^{*}-u_{m_{k}+1}\right\rangle\right] .
\end{aligned}
$$


Since $\beta_{m_{k}} \rightarrow 0$ and $\left\|u_{m_{k}}-\wp^{*}\right\|$ is a bounded, (3.29) and (3.30) imply that $\left\|u_{m_{k}+1}-\wp^{*}\right\|^{2} \rightarrow 0$ as $k \rightarrow \infty$. Further, we have $\lim _{n \rightarrow \infty}\left\|u_{k}-\wp^{*}\right\|^{2} \leq \lim _{n \rightarrow \infty}\left\|u_{m_{k}+1}-\wp^{*}\right\|^{2} \leq 0$. Hence, $u_{n} \rightarrow \wp^{*}$. This complete the proof.

From Theorem 3.1, we obtain the following result to solve variational inequalities.

Corollary 3.1. Assume that $\mathscr{L}: \mathbb{C} \rightarrow \mathbb{H}$ is a pseudomonotone, weakly continuous, and $L$ Lipschitz continuous operator and the solution set VI $(\mathscr{L}, \mathbb{C})$ is nonempty. Let $\left\{u_{n}\right\}$ be a sequence generated in the following algorithm:

Step 0: Select $\alpha \geq 0, \zeta_{1}>0, \mu \in(0,1)$, and $u_{0}, u_{1} \in \mathbb{C}$. Moreover, select a non-negative real sequence $\left\{\varphi_{n}\right\}$ such that $\sum_{n=1}^{\infty} \varphi_{n}<+\infty$, and a sequence $\left\{\beta_{n}\right\} \subset(0,1)$ such that $\lim _{n \rightarrow \infty} \beta_{n}=0$ and $\sum_{n=1}^{\infty} \beta_{n}=+\infty$.

Step 1: Compute $\chi_{n}=u_{n}+\alpha_{n}\left(u_{n}-u_{n-1}\right)-\beta_{n}\left[u_{n}+\alpha_{n}\left(u_{n}-u_{n-1}\right)\right]$, where $\left\{\alpha_{n}\right\}$ is modified on each iteration as follows:

$$
0 \leq \alpha_{n} \leq \hat{\alpha}_{n} \quad \text { and } \quad \hat{\alpha}_{n}= \begin{cases}\min \left\{\alpha, \frac{\varepsilon_{n}}{\left\|u_{n}-u_{n-1}\right\|}\right\} & \text { if } u_{n} \neq u_{n-1} \\ \alpha & \text { otherwise }\end{cases}
$$

Step 2: Compute

$$
\left\{\begin{array}{l}
y_{n}=P_{\mathbb{C}}\left(\chi_{n}-\zeta_{n} \mathscr{L}\left(\chi_{n}\right)\right), \\
u_{n+1}=P_{\mathbb{H}_{n}}\left(\chi_{n}-\zeta_{n} \mathscr{L}\left(y_{n}\right)\right),
\end{array}\right.
$$

where $\mathbb{H}_{n}=\left\{z \in \mathbb{H}:\left\langle\chi_{n}-\zeta_{n} \mathscr{L}\left(\chi_{n}\right)-y_{n}, z-y_{n}\right\rangle \leq 0\right\}$. The stepsize rule for next iteration is evaluated as follows:

$$
\zeta_{n+1}= \begin{cases}\min \left\{\zeta_{n}+\varphi_{n}, \frac{\mu\left\|\chi_{n}-y_{n}\right\|^{2}+\mu\left\|u_{n+1}-y_{n}\right\|^{2}}{2\left\langle\mathscr{L}\left(\chi_{n}\right)-\mathscr{L}\left(y_{n}\right), u_{n+1}-y_{n}\right\rangle}\right\} & \text { if }\left\langle\mathscr{L}\left(\chi_{n}\right)-\mathscr{L}\left(y_{n}\right), u_{n+1}-y_{n}\right\rangle>0 \\ \zeta_{n}+\varphi_{n} & \text { otherwise. }\end{cases}
$$

Then, $\left\{u_{n}\right\}$ converge strongly to $\wp^{*} \in V I(\mathscr{L}, \mathbb{C})$.

\section{NUMERICAL EXPERIMENTS}

In this section, we include a numerical illustration to demonstrate the validity of the proposed method. The MATLAB codes were run in MATLAB version 9.5 (R2018b) on the Intel(R) Core(TM)i5-6200 CPU PC @ 2.30GHz 2.40GHz, RAM 8.00 GB.

Problem 4.1. Let $\mathbb{C} \subset \mathbb{R}^{5}$ be defined as $\mathbb{C}:=\left\{u \in \mathbb{R}^{5}:-5 \leq u_{i} \leq 5\right\}$. Let $f: \mathbb{C} \times \mathbb{C} \rightarrow \mathbb{R}$ be defined as $f(u, y)=\langle P u+Q y+d, y-u\rangle, \forall u, y \in \mathbb{C}$, which is the Nash-Cournot equilibrium model [10]. To see the numerical efficiency of the different methods, we consider $P$ and $Q$ as follows:

$$
P=\left(\begin{array}{ccccc}
3.1 & 2 & 0 & 0 & 0 \\
2 & 3.6 & 0 & 0 & 0 \\
0 & 0 & 3.5 & 2 & 0 \\
0 & 0 & 2 & 3.3 & 0 \\
0 & 0 & 0 & 0 & 3
\end{array}\right) \quad Q=\left(\begin{array}{ccccc}
1.6 & 1 & 0 & 0 & 0 \\
1 & 1.6 & 0 & 0 & 0 \\
0 & 0 & 1.5 & 1 & 0 \\
0 & 0 & 1 & 1.5 & 0 \\
0 & 0 & 0 & 0 & 2
\end{array}\right)
$$

where $d=(1,-2,-1,2,-1)^{T}$ and Lipschitz-type constants are $c_{1}=c_{2}=\frac{1}{2}\|P-Q\|$. 
Problem 4.2. Let $\mathbb{C} \subset \mathbb{R}^{N}$ be defined as $\mathbb{C}=\left\{u \in \mathbb{R}^{N}: A u \leq b\right\}$, where $A$ is an $100 \times N$ matrix. Let $f: \mathbb{C} \times \mathbb{C} \rightarrow \mathbb{R}$ be defined as $f(u, y)=\langle\mathscr{L}(u), y-u\rangle, \forall u, y \in \mathbb{C}$, where $\mathscr{L}: \mathbb{R}^{N} \rightarrow \mathbb{R}^{N}$ is an operator defined by $\mathscr{L}(u)=P u+r$, where $r \in \mathbb{R}^{N}$ and $P=Q Q^{T}+R+S$, where $Q$ is an $N \times N$ matrix, $R$ is an $N \times N$ skew-symmetric matrix, and $S$ is an $N \times N$ positive definite diagonal matrix. It is easy to see that $f$ is monotone and the Lipschitz constants are $2 c_{1}=2 c_{2}=\|P\|$ (see [28, 29] for details).

Problem 4.3. Let $\mathbb{C} \subset \mathbb{R}^{2}$ be defined as $\mathbb{C}=\left\{u \in \mathbb{R}^{2}:\left(u_{1}-2\right)^{2}+\left(u_{2}-2\right)^{2} \leq 1\right\}$. Let $\mathscr{L}$ : $\mathbb{R}^{2} \rightarrow \mathbb{R}^{2}$ be defined by

$$
\mathscr{L}(u)=\left(\begin{array}{c}
0.5 u_{1} u_{2}-2 u_{2}-10^{7} \\
-4 u_{1}-0.1 u_{2}^{2}-10^{7}
\end{array}\right)
$$

We can evaluate that $\mathscr{L}$ is Lipschitz continuous with $L=5$, and pseudomonotone. Assume that $f(u, y)=\langle\mathscr{L}(u), y-u\rangle$, and $c_{1}=c_{2}=\frac{5}{2}$.

Problem 4.4. Let $\mathbb{H}=L^{2}([0,1])$ be a Hilbert space with inner product $\langle u, y\rangle=\int_{0}^{1} u(t) y(t) d t$, for all $u, y \in \mathbb{H}$ and induced norm $\|u\|=\sqrt{\int_{0}^{1}|u(t)|^{2} d t}$. Let $f: \mathbb{C} \times \mathbb{C} \rightarrow \mathbb{R}$ be defined by $f(u, y)=\langle\mathscr{L}(u), y-u\rangle, \forall u, y \in \mathbb{C}$, where $\mathbb{C}:=\left\{u \in L^{2}([0,1]):\|u\| \leq 1\right\}$. Moreover, assume that $\mathscr{L}: \mathbb{C} \rightarrow \mathbb{H}$ is defined by

$$
\mathscr{L}(u)(t)=\int_{0}^{1}[u(t)-H(t, s) f(u(s))] d s+g(t),
$$

where

$$
H(t, s)=\frac{2 t s e^{(t+s)}}{e \sqrt{e^{2}-1}}, \quad f(u)=\cos (u) \quad \text { and } \quad g(t)=\frac{2 t e^{t}}{e \sqrt{e^{2}-1}} .
$$

From [30], we see that $f$ is monotone (hence pseudomonotone) with Lipschitz constants $2 c_{1}=$ $2 c_{1}=2$, and the solution set is nonempty.

Now, we use Problem 4.1 to compare the numerical efficiency of Algorithm 3.1, Algorithm 3.2 in [31], Algorithm 4.1 in [11], and Algorithm 3 in [12] by taking the different initials of $u_{1}=y_{0}$ and the fixed values of $u_{0}=y_{-1}=(0,0,0,0,0)^{T}$. We can see how these initials affect the convergence of the iterative sequence. Figures 1-4 show a number of results obtained by enabling a tolerance $10^{-5}$. Information about the control parameters are taken as follows: (1) Algorithm 3.2 in [31] (Alg3.2): $\lambda=\frac{1}{\max \left\{4 c_{1}, 4 c_{2}\right\}}, \alpha_{n}=\frac{1}{20(n+2)}, D_{n}=\left\|u_{n}-y_{n}\right\|^{2}$. (2) Algorithm 4.1 in [11] (Alg4.1): $\lambda_{0}=0.25, \mu=0.33, \alpha_{n}=\frac{1}{(n+1)^{0.5}}, D_{n}=\max \left\{\left\|u_{n+1}-y_{n}\right\|^{2},\left\|u_{n}-y_{n}\right\|^{2}\right\}$. (3) Algorithm 3 in [12] (Alg3): $\lambda=\frac{1}{\max \left\{4 c_{1}, 4 c_{2}\right\}}, \theta=0.50, \varepsilon_{n}=\frac{1}{(n+1)^{2}}, \gamma_{n}=\frac{1}{20(n+2)}, \beta_{n}=$ $\frac{7}{10}\left(1-\gamma_{n}\right), D_{n}=\left\|\chi_{n}-y_{n}\right\|^{2}$. (4) Algorithm 3.1 (Alg1): $\zeta_{1}=0.25, \mu=0.33, \alpha=0.5, \varepsilon_{n}=$ $\frac{1}{(n+1)^{2}}, \beta_{n}=\frac{1}{20(n+2)}, D_{n}=\left\|\chi_{n}-y_{n}\right\|^{2}, \varphi_{n}=\frac{100}{(n+1)^{2}}$.

Next, we use Problem 4.1 to compare the numerical efficiency of Algorithm 3.1, Algorithm 3.2 in [31], Algorithm 4.1 in [11], and Algorithm 3 in [12] by letting different tolerance values, and $u_{1}=y_{0}=(1,1,1,1,1)^{T}$ and $u_{0}=y_{-1}=(0,0,0,0,0)^{T}$. Figures 5 and 6 and the following table show a number results obtained by letting different tolerance values. Information about the control parameters are same as the in the experiment above.

Next, we use Problem 4.2 to compare the numerical effectiveness of Algorithm 3.1, Algorithm 3.2 in [31], Algorithm 4.1 in [11], and Algorithm 3 in [12] by letting different dimension 

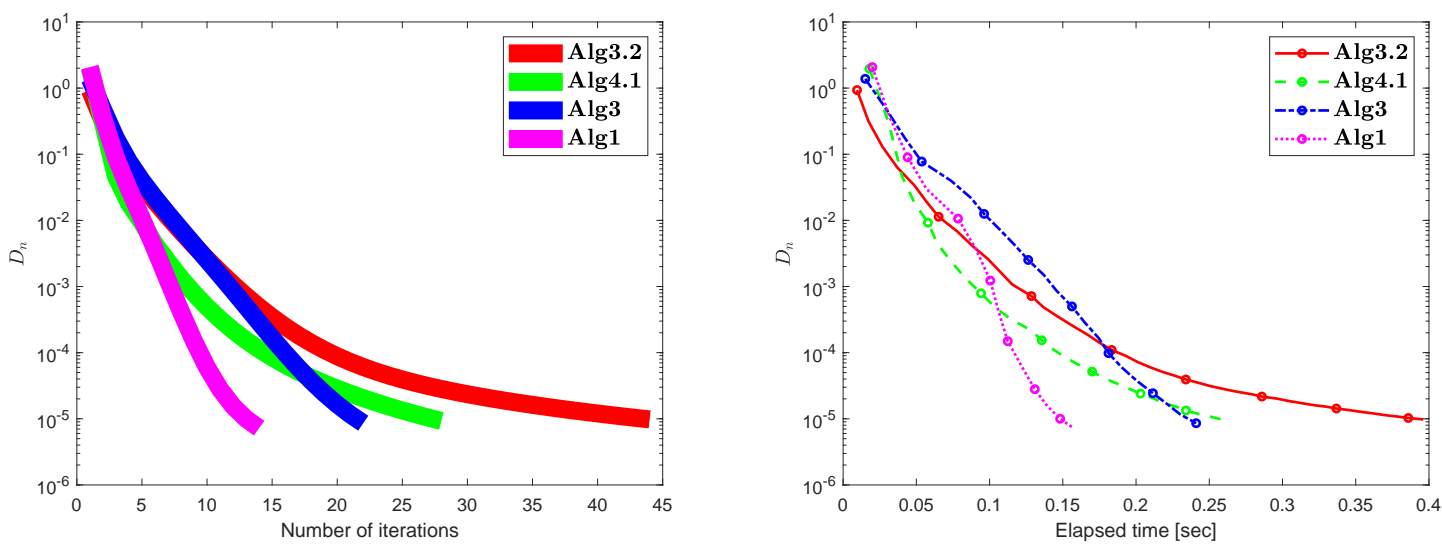

FIGURE 1. $u_{1}=(1,0,1,0,1)^{T}$ and the number of iterations are $44,28,22,14$ and elapsed time are $0.3961,0.2625,0.2410,0.1573$, respectively.
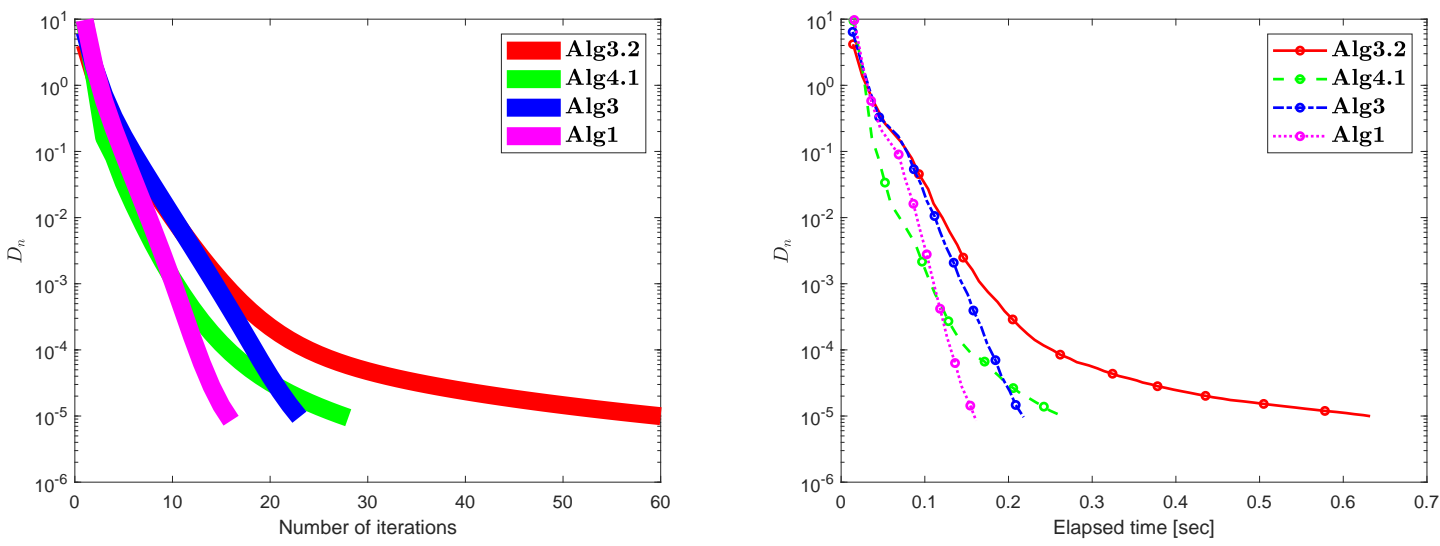

FIGURE 2. $u_{1}=(2,1,-2,1,2)^{T}$ and the number of iterations are 60, 28, 23, 16 and the elapsed time are $0.6321,0.2676,0.2180,0.1613$, respectively.

\begin{tabular}{|c|c|c|c|c|c|c|c|c|}
\hline \multirow{2}{*}{$\begin{array}{l}\text { Tolerance } \\
\text { Algo. }\end{array}$} & \multicolumn{2}{|c|}{0.01} & \multicolumn{2}{|c|}{0.001} & \multicolumn{2}{|c|}{0.0001} & \multicolumn{2}{|c|}{0.00001} \\
\hline & iter. & time & iter. & time & iter. & time & iter. & time \\
\hline Algorithm 3.2 in [31] & 7 & 0.07259 & 12 & 0.12327 & 21 & 0.19863 & 55 & 0.50759 \\
\hline Algorithm 4.1 in [11] & 5 & 0.06205 & 10 & 0.10908 & 18 & 0.17806 & 30 & 0.27618 \\
\hline Algorithm 3 in [12] & 7 & 0.08036 & 12 & 0.11527 & 16 & 0.16133 & 22 & 0.21190 \\
\hline Algorithm 3.1 & 5 & 0.05530 & 8 & 0.09403 & 10 & 0.11141 & 14 & 0.14207 \\
\hline
\end{tabular}

$N$, and $u_{1}=y_{0}=(1,1,1,1,1)^{T}$ and $u_{0}=y_{-1}=(0,0,0,0,0)^{T}$. Figure 7 show a number of results obtained by letting a tolerance $10^{-4}$. Information about the control parameters is considered as follows: (1) Algorithm 3.2 in [31] (Alg3.2): $\lambda=\frac{1}{\max \left\{3 c_{1}, 3 c_{2}\right\}}, \alpha_{n}=\frac{1}{10(n+2)}, D_{n}=\left\|u_{n}-y_{n}\right\|^{2}$. (2) Algorithm 4.1 in [11] (Alg4.1): $\lambda_{0}=0.50, \mu=0.40, \alpha_{n}=\frac{1}{(n+1)^{0.5}}, D_{n}=\max \left\{\| u_{n+1}-\right.$ $\left.y_{n}\left\|^{2},\right\| u_{n}-y_{n} \|^{2}\right\}$. (3) Algorithm 3 in [12] (Alg3): $\lambda=\frac{1}{\max \left\{3 c_{1}, 3 c_{2}\right\}}, \theta=0.50, \varepsilon_{n}=\frac{1}{(n+1)^{2}}, \gamma_{n}=$ 

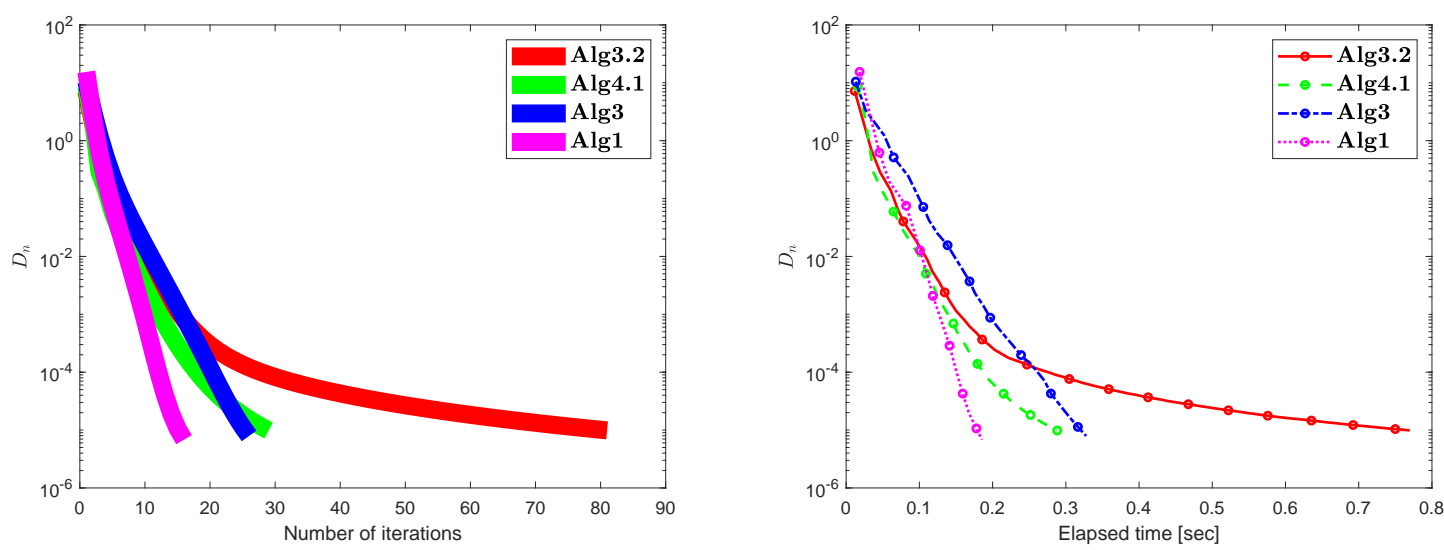

FIGURE 3. $u_{1}=(1,3,-1,2,3)^{T}$ and the number of iterations are $81,29,26,16$ and the elapsed time are $0.7697,0.2884,0.3278,0.1857$, respectively.
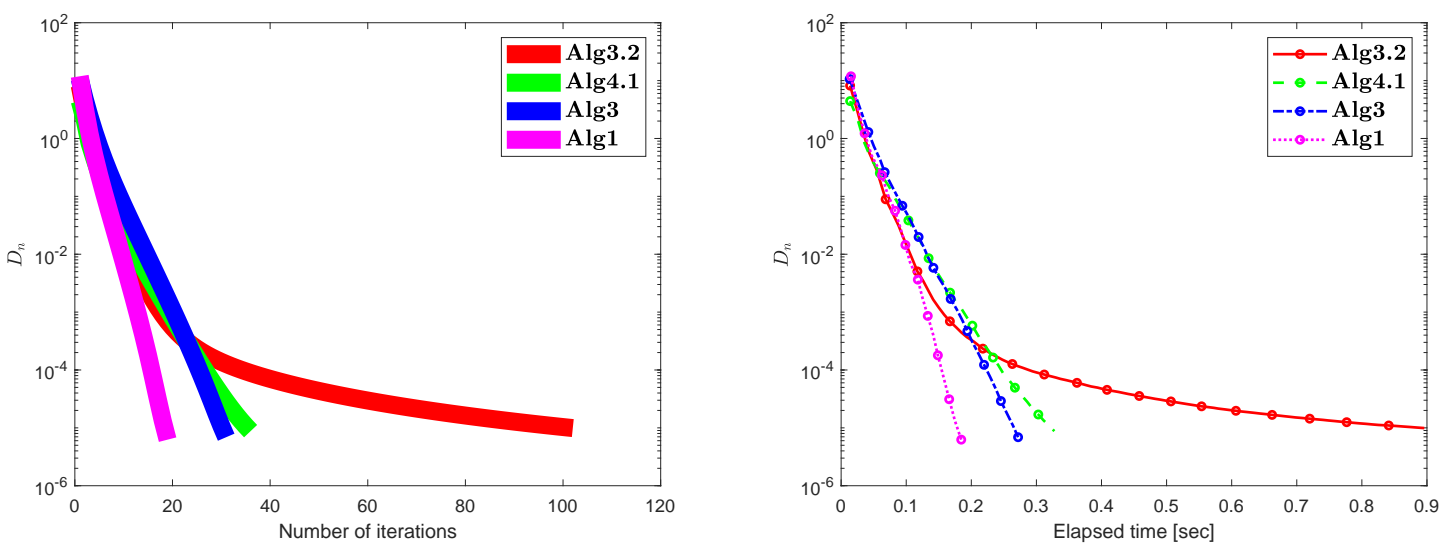

FIGURE 4. $u_{1}=(2,-1,3,-4,5)^{T}$ and the number of iterations are 102, 36, 31, 19 and the elapsed time are $0.8959,0.3275,0.2718,0.1845$, respectively.

$\frac{1}{10(n+2)}, \beta_{n}=\frac{5}{10}\left(1-\gamma_{n}\right), D_{n}=\left\|\chi_{n}-y_{n}\right\|^{2}$. (4) Algorithm 3.1 (Alg1): $\zeta_{1}=0.50, \mu=0.40, \alpha=$ $0.50, \varepsilon_{n}=\frac{1}{(n+1)^{2}}, \beta_{n}=\frac{1}{10(n+2)}, D_{n}=\left\|\chi_{n}-y_{n}\right\|^{2}, \varphi_{n}=\frac{100}{(n+1)^{2}}$.

Next, we work on Problem 4.3 to see the numerical performance of Algorithm 3.1, Algorithm 3.2 in [31], Algorithm 4.1 in [11], and Algorithm 3 in [12] by choosing the different starting points $u_{1}=y_{0}$ and the fixed values of $u_{0}=y_{-1}=(0,0)^{T}$. Figure 8 show a number of results obtain by letting a tolerance $10^{-4}$. Information about the control parameters is taken as follows: (1) Algorithm 3.2 in [31] (Alg3.2): $\lambda=\frac{1}{\max \left\{3 c_{1}, 3 c_{2}\right\}}, \alpha_{n}=\frac{1}{5(n+2)}, D_{n}=\left\|u_{n}-y_{n}\right\|^{2}$. (2) Algorithm 4.1 in [11] (Alg4.1): $\lambda_{0}=0.40, \mu=0.33, \alpha_{n}=\frac{1}{(n+1)^{0.5}}, D_{n}=\max \left\{\| u_{n+1}-\right.$ $\left.y_{n}\left\|^{2},\right\| u_{n}-y_{n} \|^{2}\right\}$. (3) Algorithm 3 in [12] (Alg3): $\lambda=\frac{1}{\max \left\{3 c_{1}, 3 c_{2}\right\}}, \theta=0.50, \varepsilon_{n}=\frac{1}{(n+1)^{2}}, \gamma_{n}=$ $\frac{1}{5(n+2)}, \beta_{n}=\frac{4}{10}\left(1-\gamma_{n}\right), D_{n}=\left\|\chi_{n}-y_{n}\right\|^{2}$. (4) Algorithm 3.1 (Alg1): $\zeta_{1}=0.40, \mu=0.33, \alpha=$ $0.50, \varepsilon_{n}=\frac{1}{(n+1)^{2}}, \beta_{n}=\frac{1}{5(n+2)}, D_{n}=\left\|\chi_{n}-y_{n}\right\|^{2}, \varphi_{n}=\frac{100}{(n+1)^{2}}$. 


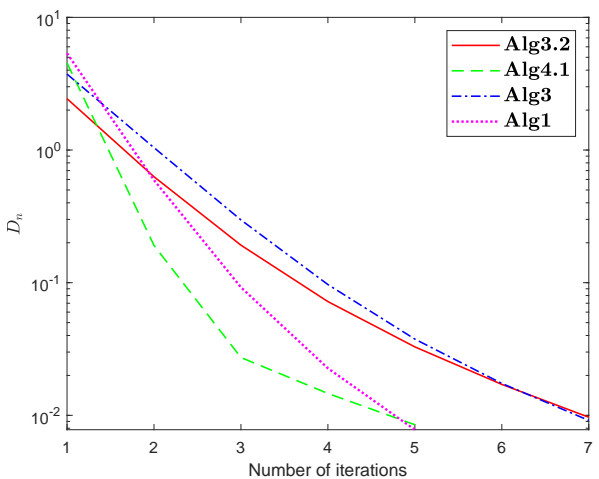

(A) tolerance $=10^{-2}$.

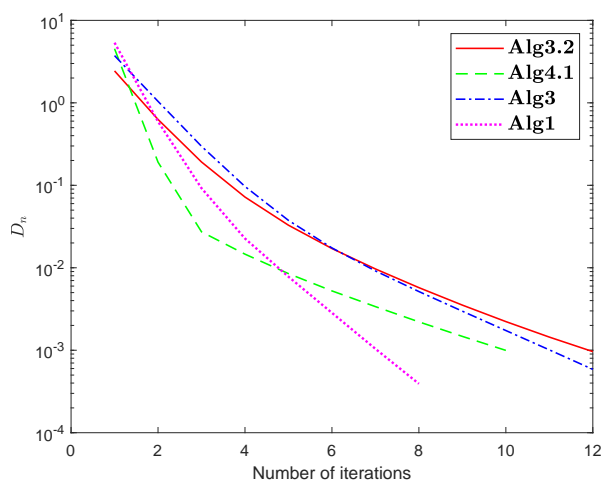

(B) tolerance $=10^{-3}$.

FIGURE 5

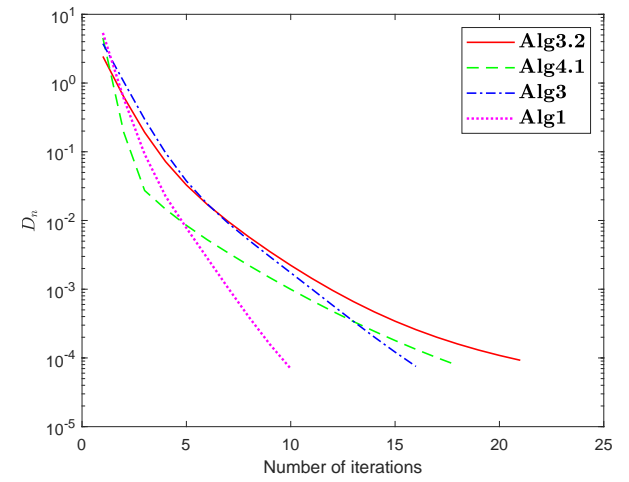

(A) tolerance $=10^{-4}$.

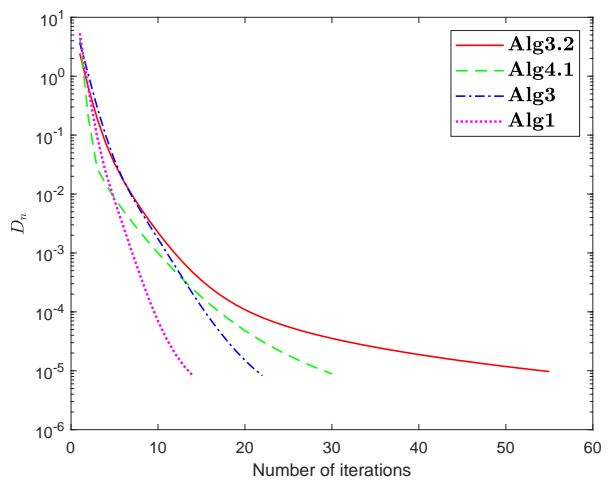

(B) tolerance $=10^{-5}$.

FIGURE 6
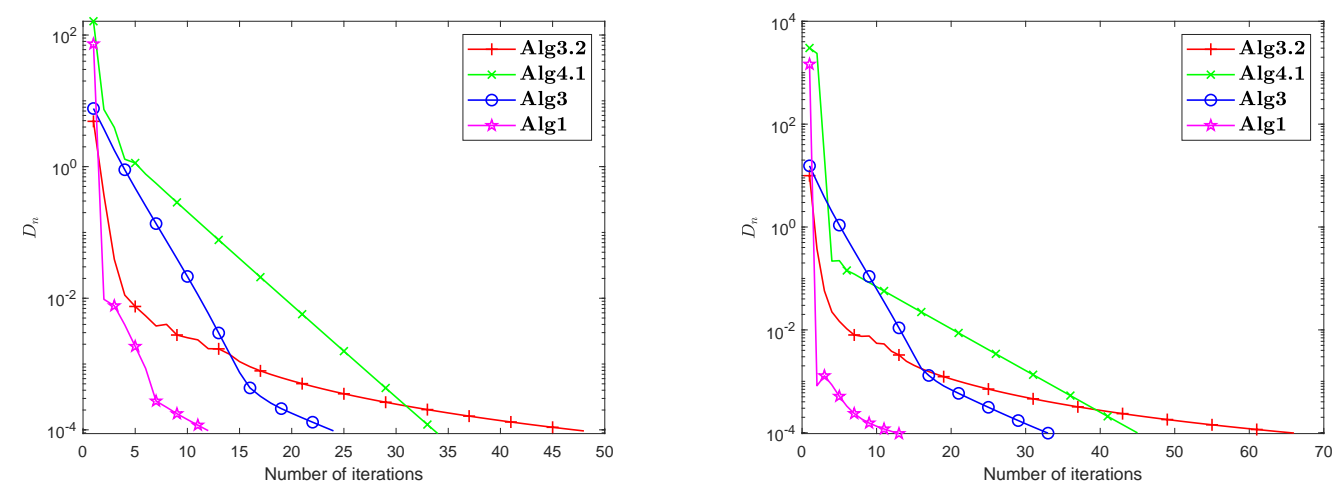

FIgURE 7. $N=5, N=10$, and the number of iterations are 48, 34, 24, 12 and $66,45,33,13$ respectively.

Finally, we work on Problem 4.4 to see the numerical effectiveness of Algorithm 3.1, Algorithm 3.2 in [31], Algorithm 4.1 in [11], and Algorithm 3 in [12] by choosing the different starting points of $u_{1}=y_{0}$, and the fixed values of $u_{0}=y_{-1}=t$. Figure 9 show a number of results 

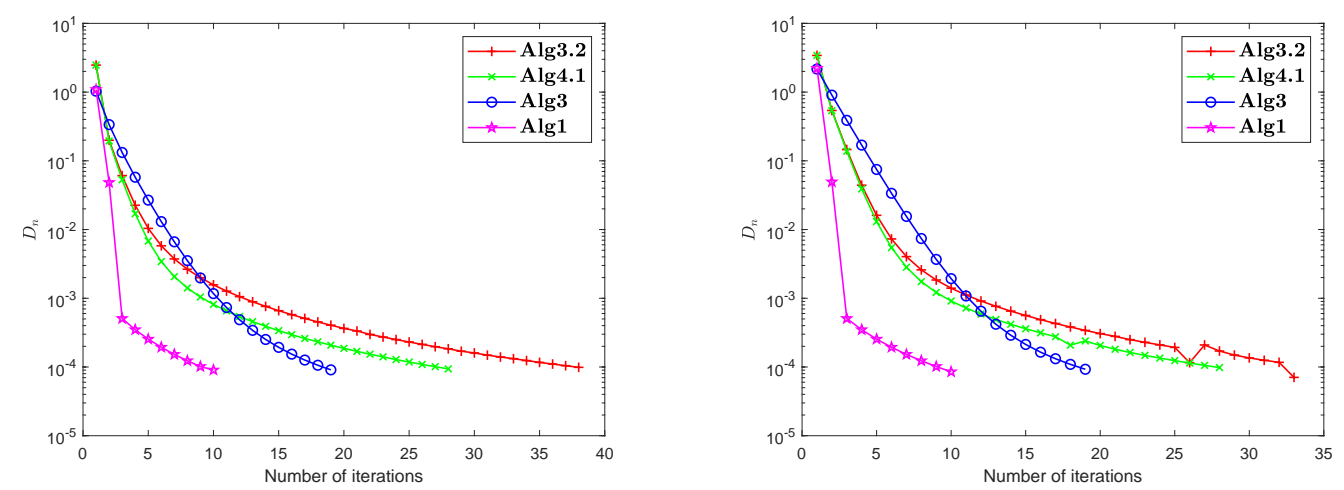

FIGURE 8. Numerical comparison when $u_{1}=(1.5,1.7)^{T}, u_{1}=(1.0,2.0)^{T}$ and the number of iterations are $38,28,19,10$ and $33,28,19,10$, respectively.

achieved by setting up a tolerance $10^{-4}$. Information about the control parameters is considered as follows: (1) Algorithm 3.2 in [31] (Alg3.2): $\lambda=\frac{1}{\max \left\{5 c_{1}, 5 c_{2}\right\}}, \alpha_{n}=\frac{1}{5(n+2)}, D_{n}=\left\|u_{n}-y_{n}\right\|^{2}$. (2) Algorithm 4.1 in [11] (Alg4.1): $\lambda_{0}=0.40, \mu=0.33, \alpha_{n}=\frac{1}{(n+1)^{0.5}}, D_{n}=\max \left\{\| u_{n+1}-\right.$ $\left.y_{n}\left\|^{2},\right\| u_{n}-y_{n} \|^{2}\right\}$. (3) Algorithm 3 in [12] (Alg3): $\lambda=\frac{1}{\max \left\{5 c_{1}, 5 c_{2}\right\}}, \theta=0.50, \varepsilon_{n}=\frac{1}{(n+1)^{2}}, \gamma_{n}=$ $\frac{1}{5(n+2)}, \beta_{n}=\frac{4}{10}\left(1-\gamma_{n}\right), D_{n}=\left\|\chi_{n}-y_{n}\right\|^{2}$. (4) Algorithm 3.1 (Alg1): $\zeta_{1}=0.40, \mu=0.33, \alpha=$ $0.50, \varepsilon_{n}=\frac{1}{(n+1)^{2}}, \beta_{n}=\frac{1}{5(n+2)}, D_{n}=\left\|\chi_{n}-y_{n}\right\|^{2}, \varphi_{n}=\frac{100}{(n+1)^{2}}$.
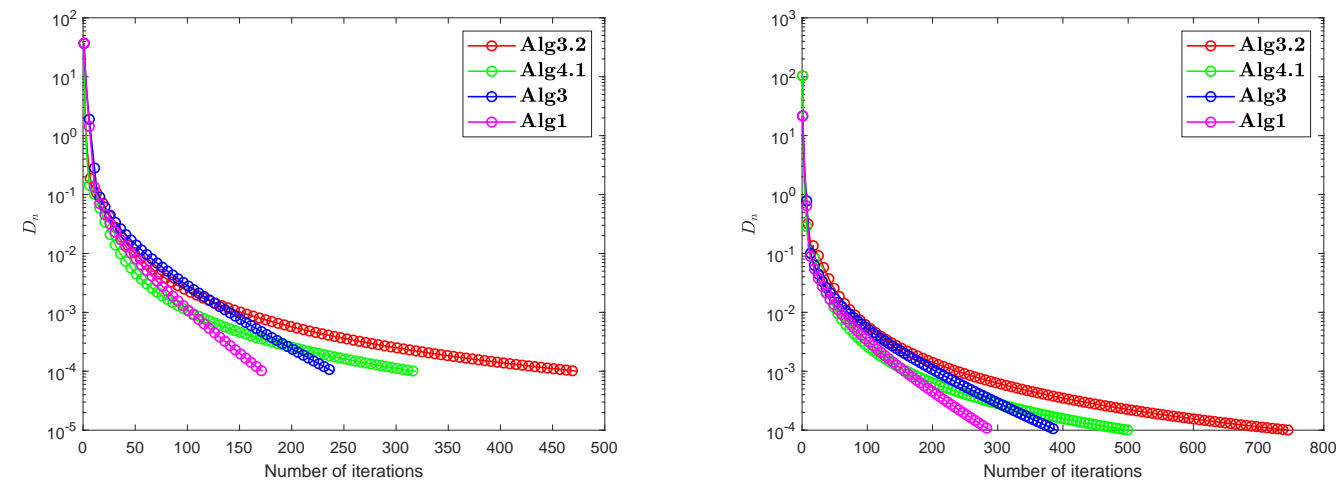

FIGURE 9. Numerical efficiency comparison when $u_{1}=\left(t^{3}-2 t+4\right) e^{t}, u_{1}=$ $\left(3 t^{2}-1\right) \cos (t)$ and the number of iterations are 474, 319, 240, 172 and 747, $500,390,288$, respectively.

\section{Acknowledgements}

The authors acknowledge the financial support provided by the Center of Excellence in Theoretical and Computational Science (TaCS-CoE), KMUTT. This project was funded by National Council of Thailand (NRCT) under Research Grants for Talented Mid-Career Researchers (Contract No. N41A640089). 


\section{REFERENCES}

[1] L.D. Muu, W. Oettli, Convergence of an adaptive penalty scheme for finding constrained equilibria, Nonlinear Anal. 18 (1992), 1159-1166.

[2] E. Blum, W. Oettli, From optimization and variational inequalities to equilibrium problems, Math. Student 63 (1994), 123-145.

[3] S.Y. Cho, A monotone Bregan projection algorithm for fixed point and equilibrium problems in a reflexive Banach space, Filomat, 34 (2020), 1487-1497.

[4] L.V. Nguyen, Q.H. Ansari, X. Qin, Weak sharpness and finite convergence for solutions of nonsmooth variational inequalities in Hilbert spaces, Appl. Math. Optim. 84 (2021), 807-828.

[5] L.C. Ceng, A subgradient-extragradient method for bilevel equilibrium problems with the constraints of variational inclusion systems and fixed point problems, Commun. Optim. Theory, 2021 (2021), Article ID 4.

[6] K. Fan, A minimax inequality and applications, Inequalities III (O. Shisha, ed.). Academic Press, New York, 1972.

[7] P. Sunthrayuth, H.U. Rehman, P. Kumam, A modified Popov's subgradient extragradient method for variational inequalities in Banach spaces, J. Nonlinear Funct. Anal. 2021 (2021), Article ID 7.

[8] S.Y. Cho, X. Qin, On the strong convergence of an iterative process for asymptotically strict pseudocontractions and equilibrium problems, Appl. Math. Comput. 235 (2014), 430-438.

[9] T.D. Quoc, P.N. Anh, L.D. Muu, Dual extragradient algorithms extended to equilibrium problems, J. Global Optim. 52 (2011), 139-159.

[10] D.Q. Tran, M.L. Dung, V.H. Nguyen, Extragradient algorithms extended to equilibrium problems, Optimization, 57 (2008), 749-776.

[11] D.V. Hieu, J.J. Strodiot, L.D. Muu, Strongly convergent algorithms by using new adaptive regularization parameter for equilibrium problems, J. Comput. Appl. Math. 376 (2020), 112844.

[12] N.T. Vinh, L.D. Muu, Inertial extragradient algorithms for solving equilibrium problems, Acta Math. Vietnamica 44 (2019), 639-663.

[13] S.D. Flåm, A.S. Antipin, Equilibrium programming using proximal-like algorithms, Math. Program. 78 (1996), 29-41.

[14] G.M. Korpelevich, The extragradient method for finding saddle points and other problems, Matecon, 12 (1976), 747-756.

[15] L. Liu, B. Tan, S.Y. Cho, On the resolution of variational inequality problems with a double-hierarchical structure, J. Nonlinear Convex Anal. 21 (2020), 377-386.

[16] H. Rehman, P. Kumam, A.B. Abubakar, Y.J. Cho, The extragradient algorithm with inertial effects extended to equilibrium problems, Comput. Appl. Math. 39 (2020), 100.

[17] H. Rehman, P. Kumam, Y.J. Cho, Y.I. Suleiman, W. Kumam, Modified popov's explicit iterative algorithms for solving pseudomonotone equilibrium problems, Optim. Meth. Softw. 36 (2021), 82-113.

[18] H. Rehman, P. Kumam, Y.J. Cho, P. Yordsorn, Weak convergence of explicit extragradient algorithms for solving equilibirum problems, J. Inequal. Appl. 2019 (2019), 282.

[19] B. Tan, S.Y. Cho, Strong convergence of inertial forward-backward methods for solving monotone inclusions, Appl. Anal. (2021), 10.1080/00036811.2021.1892080.

[20] B.T. Polyak. Some methods of speeding up the convergence of iteration methods, USSR Comput. Math. Math. Phys. 4 (1964), 1-17.

[21] F. Alvarez, H. Attouch, An inertial proximal method for maximal monotone operators via discretization of a nonlinear oscillator with damping, Set-Valued Var. Anal. 9 (2001), 3-11.

[22] A. Beck, M. Teboulle, A fast iterative shrinkage-thresholding algorithm for linear inverse problems, SIAM J. Imaging Sci. 2 (2009), 183-202.

[23] Y. Censor, A. Gibali, S. Reich, The subgradient extragradient method for solving variational inequalities in hilbert space, J. Optim. Theory Appl. 148 (2010), 2010.

[24] M. Bianchi, S. Schaible, Generalized monotone bifunctions and equilibrium problems, J. Optim. Theory Appl. 90 (1996), 31-43.

[25] J.V. Tiel, Convex Analysis: An Introductory Text, 1 edition, Wiley, New York, 1984. 
[26] H.K. Xu, Another control condition in an iterative method for nonexpansive mappings, Bull. Aust. Math. Sco. 65 (2002), 109-113.

[27] P.E. Maingé, Strong convergence of projected subgradient methods for nonsmooth and nonstrictly convex minimization, Set-Valued Anal. 16 (2008), 899-912.

[28] M.V. Solodov, B.F. Svaiter, A new projection method for variational inequality problems, SIAM J. Control Optim. 37 (1999), 765-776.

[29] Q.L. Dong, Y.J. Cho, L.L. Zhong, T.M. Rassias, Inertial projection and contraction algorithms for variational inequalities, J. Global Optim. 70 (2017), 687-704.

[30] D.V. Hieu, P.K. Anh, L.D. Muu, Modified hybrid projection methods for finding common solutions to variational inequality problems, Comput. Optim. Appl. 66 (2016), 75-96.

[31] D.V. Hieu, Halpern subgradient extragradient method extended to equilibrium problems, Rev. R. Acad. Cienc. Exactas Fis. Nat. Ser. A Math. RACSAM 111 (2016), 823-840. 